\title{
John Tooby y Leda Cosmides: psicología evolutiva y falsa interdisciplina*
}

John Tooby and Leda Cosmides: Evolutionary Psychology and the Mismeasure of Interdiscipline

\author{
Julio Muñoz Rubio ${ }^{\dagger \ddagger}$
}

\begin{abstract}
Resumen
El término "interdisciplina" se ha venido siendo usado con creciente frecuencia. Una adecuada caracterización de ella debe contener un análisis histórico del origen y desarrollo de las disciplinas. La disciplinarización del conocimiento ha expresado históricamente un criterio epistemológico eficientista, mecanicista e ideológico. Estos criterios se pueden reproducir en la construcción de interdisciplinas fallidas, en particular en las ciencias de la vida.

En teoría de la evolución, la psicología evolutiva es ejemplo de interdisciplina fallida, en especial con la obra de Tooby y Cosmides. Estos autores intentan fusionar ramas de la biología previamente existentes en una nueva explicación sobre la conducta humana, pero su intento falla al no aplicar una concepción relacional y en cambio mantener una concepción esencialista. Insisten en una metodología que asigna a una unidad biológica y genética fundamental las propiedades que han de expresarse en todos los niveles de análisis, terminando por el conductual y el cultural, mediante procesos de transmisión unidireccional de información. Con ello, su pretendida interdisciplina se pierde en favor de otra re-disciplinarización, reduccionista e ideológica.

Se considera que la construcción de una biología interdisciplinaria debe despojarse de estas ideológías, entendiendo ideología como "falsa conciencia" desde su acepción marxista; se debe, así, considerar a las disciplinas dialécticamente como campos de transición interpenetrados, no esencialistas y sin jerarquías análiticas y ontológicas pre-construidas.
\end{abstract}

Palabras clave: interdisciplina - evolución - dialéctica - ideología - psicología evolutiva

\footnotetext{
Abstract

The term "interdiscipline" has been coming into use with increasing frequency. An accurate characterization of this term must contain a historical analyses regarding the origins and development of disciplines. Historically speaking, disciplinarization of knowledge has been a result of efficientist, and ideological criteria, all of which might be reproduced in the construction of false interdisciplines, particularly in life sciences.

Evolutionary Psychology is an example of such a false interdiscipline in theory of evolution. This is particularly clear in the works by Tooby and Cosmides. These authors, attempt to fuse some previously existing branches of biology, in order to build a new explanation about human behavior. But their attempt fails as they lack of a relational conception and, instead, they put into practice an essentialist methodology that ascribe to a fundamental biological and genetic unity, the properties that will express in all the levels of analyses including the behavioral and cultural ones. This is conceived as occurring by means of a one-directional transmission of information processes. Due to this, their so-pretended interdisciplinarity, actually becomes another ideological and reductionist re-disciplinarization.

The construction of an interdisciplinary biology must get rid of these ideologies, considering disciplines as dialectical interpenetrated transitions, without rigid essences, and without ontological and predetermined analytical hierarchies.

Keywords: interdiscipline - evolution-dialectics - ideology - evolutionary psychology

${ }^{*}$ Recibido: 29 de Marzo de 2016. Aceptado con revisiones: 5 de Diciembre de 2016.

${ }^{\dagger}$ Centro de Investigaciones Interdisciplinarias en Ciencias y Humanidades, Universidad Nacional Autónoma de México. Para contactar al autor, por favor, escribir a: juliomunozr2000@yahoo.es.

* Se agradece a la Dirección General de Asuntos del Personal Académico de la Universidad Nacional Autónoma de México por su apoyo a través del proyecto IN 400416.

Metatheoria 8(2)(2018): 87-96. ISSN 1853-2322.

(c) Editorial de la Universidad Nacional de Tres de Febrero. Publicado en la República Argentina.
} 


\section{Interdisciplina y falsas interdisciplinas}

A grandes rasgos, en la imposibilidad de dar una definición definitiva de interdsiciplina podemos decir que se trata de un trabajo teórico-práctico en el que se funden y asimilan teorías, lenguajes y prácticas provenientes de distintos campos de conocimiento, en una propuesta o un cuerpo de conocimiento nuevos, con un lenguaje y metodologías propias, que intentan superar el de cada uno de sus antecesores. Algunos ejemplos claros de estas fusiones en ciencia biológica se adivinan desde su nombre, bioquímica, biofísica, ecología conductual y otros menos evidentes se encuentran en la ecología de poblaciones, genética de poblaciones, que utilizan las matemáticas en campos aplicados a las relaciones entre organismos o la dinámica hereditaria. Algunos de los más importantes proponentes y analistas de la interdisciplina son, Klein (1990, 1996), Szosteck (2010), Kockelmans (1979), Fonkenthal (2001), Strober (2011), Weingart y Stehr (2000), Newell (1990), Palmer (2000), Frodman et al. (2010), Repko (2014), Farrel et al. (2013), Ostreng (2010).

La interdisciplina no es mezcla ecléctica ni suma de conocimientos previos, sino forma cualitativamente diferente de ellos, que puede explicar más de lo que anteriormente se podía hacer; comprender aquello que, por causa de la parcelación del conocimiento previo no era posible entender.

La interdisciplina surge ante una dificultad creciente de desarrollar la ciencia con una separación permanente entre los campos de conocimiento. La ciencia creció durante varios siglos mediante este proceso de especialización y ramificación, con raíces cartesianas, que le fue muy fructífero. Es aproximadamente a mediados del siglo XIX que esta tendencia cambia como resultado de los trabajos de Darwin, quien integra conocimientos provenientes de geología, paleontología, economía política, taxonomía (animal y vegetal), psicología, cuando menos, para dar una explicación espacio-temporal de la vida en la Tierra; de Marx-Engels en un modelo donde lo filosófico, la historia la economía y la teoría del Estado quedan integradas en una explicación de la dinámica social. Décadas más tarde el desarrollo del psicoanálisis freudiano y las interpenetraciones entre yo-super yo-ello, consciente, preconsciente e inconsciente y la disyuntiva principio de realidad-principio de placer. Estas aportaciones marcan una nueva era en la ciencia al poner el acento en fenómenos y sistemas de estudio caracterizados por su complejidad, por la concurrencia de una cantidad de variables simultáneas que desafían al modelo binario, variable independiente-variable dependiente característico de los sistemas simples, utilizados en la física y la química clásicas.

Tenemos, pues, que tanto el conocimiento disciplinario como el interdisciplinario son productos históricos y no resultados de una tendencia "objetiva" ni mucho menos "inevitable" para expresar una naturalidad en el ordenamiento del mundo.

Uno de los problemas que se manifiesta con el desarrollo de los estudios de la complejidad y la interdisciplina, es que frecuentemente se revisten de contenidos, conceptos y metodologías transportados mecánicamente desde la ciencia disciplinar. Eso limita su desarrollo. No se comprende que la interdisciplina (y la transdisciplina) pertenece a formas cualitativamente distintas de conocimiento, con metodologías y conceptos propios.

Este proceder lleva a la construcción de lo que podemos calificar como "falsas interdisciplinas", Tales serían los casos de a) aquella que eterniza y naturaliza la existencia de las disciplinas tal y como las conocemos, con sus fronteras bien delimitadas y fijas y b) la que parta de la existencia de disciplinas como resultados inevitables del progreso social y científico. Estas dos formas interdisciplinarias son falsas porque al defender la existencia de un mundo disciplinarizado, se obstaculiza el movimiento del conocimiento en planos horizontales, que hagan transiciones entre las disciplinas al punto de borrar su existencia como algo permanente. Categorías de análisis y conceptos deben tener como condición la interdefinibilidad (García 2007, pp. 143-144) en los distintos campos que armen a la interdisciplina, tendiendo a borrar las fronteras entre ellos.

En otras palabras, la disciplinarización forzada del mundo científico es un componente importante de falsas conciencias. 
Otra forma de falsa interdisciplina es aquella que se construye de modo que deliberadamente deja fuera de su estudio a los campos del conocimiento cuya aplicación o inserción en el modelo interdisciplinario invalidaría los pilares de la teoría construida. Aquí lo que ocurrirá, es que se construye una interdisciplina que afirma que va a mostrar un camino hacia la totalidad cuando en realidad deja de lado la totalidad por medio de la exclusión deliberada de aquello que no conviene que se incluya.

Finalmente, la cuarta forma pseudo-interdisciplinaria, que centrará nuestra atención en este trabajo es aquella que mantiene las visiones esencialista y monistas del mundo, es decir, la interdisciplina que, a pesar de poner énfasis y aceptar la complejidad de los de los procesos del universo que ella estudia, termina analizándolos en referencia a una causa última o esencial que se reproduce y expresa en todos los niveles del sistema bajo estudio, transmitiendo a todos ellos la supuesta propiedad esencial que debe comprenderse.

Esta intentona pseudo-interdisciplinaria, esencialista y reduccionista proviene de una concepción fisicalizada, en la que se busca la raíz expresable en magnitudes, de cualquier rama de la ciencia, bajo la justificación de que el lenguaje de la física posee un carácter unificador. Es así como Carnap (1965), defiende la posibilidad de expresar la psicología en ese lenguaje de magnitudes y Neurath (1965) hace lo propio en el caso de la sociología.

Aquí se indica una tenencia a unificar las disciplinas al mismo tiempo que desarrollarlas, pero el problema que presentan muchos de los intentos de síntesis y unificación del conocimiento, es que no consideran la existencia ni de distintos niveles de análisis en el sistema bajo estudio, ni de la autonomía de estos entre sí. Tampoco las síntesis resultado de la oposición de los elementos que intervienen en el sistema. Al sistema se le observa como mero trasmisor de información generada en un nivel considerado "básico". Y a los enunciados que lo describen, como una forma lingüística de describir tales procesos de transmisión. Una interdisciplina así, es una concurrencia de saberes para construir versiones esencialistas disfrazadas de integradoras, y retornar al saber fragmentado tradicional, de remitir todo el conocimiento generado en esta concurrencia a una sola explicación.

\section{Interdisciplina e ideología}

Para ilustrar el carácter de las metodologías interdisciplinarias falsas, y en especial las de ese corte reduccionista, considero pertinente analizar el concepto marxista de ideología.

De acuerdo con el punto de vista de Engels ([1893] 1997), la ideología es un proceso mental consistente en una inversión de la relación sujeto-objeto. En este proceso, el sujeto deja impreso en el objeto y en otros sujetos cognoscentes, la idea particular que tiene sobre el objeto. Es una imposición de una idea preconcebida al objeto bajo estudio; un proceso en el que el pensamiento se eleva por encima de la realidad y se independiza de ella en actos inconscientes en los que parece que la idea corresponde enteramente a la realidad sin que el sujeto se aperciba de la forma en la que en rigor, le impone a la realidad externa su idea de cómo es. Aquí, la mente humana da la ilusión de existir separada de sus relaciones; parece adquirir un movimiento propio en el que el pensamiento no requeriría más que de sí mismo para producirse y expresar la realidad fielmente. Esto obstaculiza el camino a la objetividad y la verdad. Esta tesis general ha sido aceptada, en su forma más general, por el pensamiento marxista, a pesar de sus diferencias internas (Lukacs [1923] 1969, Hahn 2000, Milic 2000, Althusser 1988, Ranciêre 1994, Zizek 1992)

La ideología no es, pues, algo tangible, objetivable, medible; se trata de un conjunto de ideas presentadas como reflejo o explicación de una realidad con toda su "pureza", la cual sería a su vez, resultado de la "pureza" del pensamiento objetivo. Esa es la apariencia de naturalidad con la que la ideología se reviste. El individuo construye la ilusión de una "pura y desnuda" comprensión de la realidad misma, cuando lo que en realidad logra es tomar una percepción marcada por la imposición 
ideológica creyendo falsamente que esa percepción no es sino la de las cosas tal cuales son (Althusser 1988, p. 51). Así la conciencia del sujeto se troca en conciencia falsa.

La consecuencia de esto es la inversión de las relaciones abstracto-concreto, en la que el sujeto pensante analiza y conoce una realidad concreta, la realidad correspondiente a un contexto o período y llega a pensar en ella como si fuera toda la realidad, como algo supra social y ahistórico.

Marx ([1845]1953) estableció que en la medida en que este proceso se auto-produce dentro de la estructura social de clases, produce visiones del mundo y consensos construidos generalmente alrededor de las ideas de la clase dominante; genera la ilusión de ser independientemente de la voluntad de las personas, algo que impele a los sujetos a comprender el mundo y a comportarse con arreglo a principios externos al ser humano, como si le preexistieran.

El sujeto que vive arraigado a esta falsa conciencia se ve tan embebido en ella que es él mismo el que construye una falsa realidad, en este sentido, es el individuo el que es ideológico en la medida de que no sólo produce sino vive dentro de la realidad falsa, obligando a aceptarla (Zizek 1992, pp. 338339).

Kosik (1967) explica que, en el capitalismo, quienes deciden acerca de las condiciones sociales pueden sentirse conformes mientras se construyan dentro de un mundo de lo que llama "formas fenoménicas, sustraídas a su concatenación interna y completamente incomprensibles en este asilamiento" (p. 26). Menciona que existe un conjunto de fenómenos cotidianos interiorizados en las conciencias en este modo inconexo y que forman "el mundo de la pseudoconcreción", entre ellos se encuentran,

El mundo de las representaciones comunes, que son una proyección de los fenómenos externos en la conciencia de los hombres, producto de la práctica fetichizada y forma ideológica de su movimiento; -el mundo de los objetos fijados, que dan impresión de ser condiciones naturales, y no son inmediatamente reconocidos como resultado de la actividad social de los hombres (Kosik 1967, p. 27).

La ciencia ocupa un lugar preponderante en el desarrollo de esas "representaciones comunes" y de esos "objetos fijados". Con su autoridad de pretendida neutralidad y objetividad, tiene la función de construir consensos sociales y subsecuentemente convertir sus conclusiones en saberes que se proyectan a la comunidad como si fueran mero "sentido común" y por tanto no requieren de mayor corroboración ni están sujetas a falsación. Al interior de sus comunidades utiliza símbolos, objetos y categorías de análisis como si fueran manifestación de las condiciones más naturales de trabajo.

Para Kosik es la comprensión de la totalidad concreta lo que conduce a la destrucción de la pseudo concreción y con ello de la realidad fetichizada e ideologizada (1967, pp. 73-74) y a comprender como historia, como determinaciones concretas y como devenir a los fenómenos estudiados; a romper las inconexas "formas fenoménicas" y a reconstruir y recrear la variedad del mundo, la cual ha sido "arrojada al abismo de la sustancia inmutable" (1967, p. 32). Este es un agudo argumento dirigido contra el esencialismo, pues al perder la conexión con la totalidad y ofrecer sólo alícuotas de realidades inconexas, trastoca la sustancialidad del mundo que pretende mostrar. Lo que imagina como esencial resulta ser superficialidad e inmediatez, pero esta curiosa inversión no puede comprenderse ni revertirse si no es por medio del análisis de la totalidad.

La interdisciplina que no se dirija a la construcción de la totalidad y/o que permanezca encerrada en esencialismos e inmutabilidades, es ideologizada y falsa. Aquí interviene el problema de las relaciones ciencia- ideología. Dentro del marxismo la discusión acerca de esta relación ha sido muy larga y compleja, desde quien plantea su carácter excluyente per se (Althusser 1988) hasta quien niega la posibilidad de separarlas (Young 1973). Por mi parte, rechazo ambas posiciones y afirmo que la presencia de la ideología en la ciencia es un proceso complejo y flexible, en el que las condiciones sociales en un sentido muy amplio, son las que producen, en diversas proporciones, la intromisión de la primera en la segunda, pero en una sociedad libre, es decir una sociedad sin clases, es de esperarse que la ideología, en la medida en que en la vida social misma ya no tenga un peso importante, logre separarse de la ciencia. 


\section{La psicología evolutiva como interdisciplina fallida}

$\mathrm{Al}$ interior de la biología evolutiva, se producen y reproducen tensiones y colisiones entre lo que son visiones interdisciplinarias ideologizadas y las que intentan desprenderse de ese lastre.

En los intentos por analizar la naturaleza humana con el enfoque de la evolución es donde es más claro el desarrollo de las llamadas pseudociencias del determinismo biológico, tales como la craneometría y la criminología lombrosiana en la transición del siglo XIX al XX; los estudios sobre el coeficiente intelectual a partir de las primeras décadas del siglo XX, la etología humana desde los años 50 y la sociobiología desde la década de los 70's (Gould 1981, Lewontin, Rose \& Kamin 1984, Pichot 2000). La sociobiología y la psicología evolutiva, que nacen con un espíritu pretendidamente interdisciplinario y totalizador, nos brindan la oportunidad de comprender las deficiencias para constituirse en una interdisciplina sólida.

Edward O. Wilson elaboró su sociobiología haciendo una integración de la genética de poblaciones, la biología molecular, la etología y la genética conductual $(1975$, p. 5). En esta obra, que ya en su título anuncia un intento de síntesis y totalización, y en la escrita años después y dedicada exclusivamente al ser humano (Wilson 1978), el autor es claro, Todas las características de los seres sociales están en estricta determinación genética. Los ámbitos distintos en los que se expresan los genes difieren, pero el origen de unas y otras características es común y hay que explicarlas reduciéndolas a su esencia.

Posteriormente, Lumsden y Wilson $(1981,1983)$ elaboran una teoría epigenética para explicar la conducta humana. En ella, las unidades de transmisión cultural, los genes culturales o culturgenes, son entendidos en interacción con los genes biológicos y dando lugar a los hábitos culturales, pero tal interacción está fijamente jerarquizada. Los autores postulan que sólo el cambio en las frecuencias génicas puede dar como consecuencia un cambio en los genes culturales (Lumsden \& Wilson 1981, pp. 172, 272, 289), éstos siguen determinados por lo genético, como en el caso del miedo a las serpientes $(1981$, p. 83) o la evasión del incesto (1981, pp. 85-86). La interdisciplina que estos autores pretenden construir tropieza cuando se rechaza la autonomía de lo epigenético con respecto a lo genético y se le subordina a este nivel.

La psicología evolutiva surge a partir de la década de los 90 derivada de la sociobiología. Esta más reciente rama de la biología humana intenta evitar las simplificaciones de su antecesora y elabora un discurso en apariencia más multidimensional. Tooby y Cosmides, pioneros de este campo, se proponen explicar la conducta de Homo sapiens integrando conocimientos de psicología, biología evolutiva y sociología.

Desde 1992, estos autores levantan la voz criticando a la ciencia social y lo que llaman su "modelo standard" por su empeño fragmentador de dividir la conducta humana en social y biológica y no comprender la importancia de esta última. Señalan que es fundamental incorporar los componentes sociales para explicar la conducta humana, pero nunca podrá ser comprendida con independencia de su componente biológico.

Años después afirmarán,

La psicología evolutiva es el intento científico largamente anticipado para poder reunir disciplinas humanas inconexas, fragmentarias y mutuamente contradictorias, en un solo marco de investigación integrado de manera lógica tanto para las ciencias psicológicas, como para las sociales y conductuales un marco que no sólo incorpore las ciencias de la evolución sobre una base más completa y equitativa, sino que $[\ldots]$ logre resolver todas las revisiones que se hacen acerca de las creencias y las prácticas de investigación que tal síntesis requiere (Tooby \& Cosmides 2005, p. 5).

La discusión que estos psicólogos evolutivos proponen contra el sociologismo, se justifica en parte si de lo que se trata es pronunciarse contra quienes, por negar que lo esencial de las características humanas estén en la esfera de lo biológico, se van a otro extremo y trasladan su esencialismo a un ámbito 
enteramente "social" o "cultural", pero imprecisamente caracterizado o tajantemente separado de los componentes biológicos.

Sin embargo, las expectativas generadas para desarrollar un conocimiento totalizador sobre la naturaleza humana se frustran cuando la psicología evolutiva se retrotrae al discurso dominado por esencialismos, parcialidades e inmediateces derivados de las categorías de la economía política y de las nociones patriarcales sobre la conducta sexual, mediante propuestas como la teoría de inversiones de Trivers (1971).

Tooby y Cosmides (1992) muestran este proceder cuando afirman que "[l]os procesos evolutivos son el 'arquitecto' que armó, detalle a detalle, nuestra arquitectura psicológica y evolucionada" (Tooby \& Cosmides 1992, p. 50), añadiendo que "[l]os diseños complejos de estos mecanismos ["evolucionados"] son los principales canales causales a través de los cuales las ciencias naturales se conectan y moldean la sustancia de las ciencias 'sociales'” (Tooby \& Cosmides 1992, p.50).

De acuerdo con esto, son los mecanismos de la evolución biológica son los que dominarán sobre los de contenido independiente de la "arquitectura" psicológica. Lo social y lo cultural derivan de lo biológico unidireccionalmente.

Ambos autores son aún más enfáticos cuando aseveran,

[clada vez que un gen es seleccionado sobre otro, se selecciona también un diseño para un programa de desarrollo sobre otro, por virtud de su estructura, este programa de desarrollo interactúa con algunos aspectos del ambiente más que con otros interpretando ciertas características ambientales como relevantes al desarrollo (Tooby y Cosmides 1992, p. 84).

El punto es simplemente que los fenómenos sociales y culturales nunca se pueden divorciar de la estructura de la arquitectura o entendidos sin referencia a su diseño (Tooby \& Cosmides 1992, p. 122).

Estos autores siguen adelante diciendo que es la variación genética en los programas de desarrollo quien explora en el ambiente cuáles serán las fuentes informativas reguladoras del desarrollo y la conducta (Tooby \& Cosmides 2005, p. 35), es decir una epigénesis genocéntrica. Afirman que sin la presencia de las "estructuras evolucionadas", las "respuestas ambientales contingentes" no serían posibles, y que la conexión organismo-ambiente requiere de la conexión proveniente de lo “evolucionado" (Tooby \& Cosmides 2005, p. 38), que para ellos es lo genético o lo derivado directamente de él. Afirman el imperativo de comprender fenómenos como miedo, celos, atracción sexual y percepciones estéticas o de disgusto en términos computacionales (Tooby \& Cosmides 2005, p. 14), lo cual está en clara coincidencia con Pinker (1997, pp. 59-148).

En esto se expresa una deficiencia del reduccionismo en biología que lo incompatibiliza con una interdisciplinariedad no fallida, en la medida en que la interdisciplina se esfuerza por aproximarse, desde distintos ángulos, a la complejidad de los sistemas. Como es constante en el reduccionismo biologicista, las tesis de Tooby y Cosmides dibujan un sistema simple de relaciones uno a uno de causaefecto. Para ellos, el diseño para un programa de desarrollo se deriva unidireccionalmente de una selección génica por encima de otra. El gene es el agente que cambia primero y después hace que cambie el programa de desarrollo derivado del cambio en las frecuencias alélicas. De ahí derivará el cambio cultural. El deficiente mecanismo propuesto implica que la causa es una y así es el efecto, la causa es siempre la misma y tiene el mismo efecto, la causa precede al efecto siempre, y la parte precede al todo (Levins \& Lewontin 1985, pp. 267-288).

La interacción genes-ambiente que se observa con este esquema deja siempre en el lugar preponderante a los genes. El organismo es el objeto pasivo, adaptable de la evolución, el ambiente es la parte activa, el sujeto del cambio. La existencia de uno es independiente de la del otro (Lewontin 1985, pp. 85-106, 2000, pp. 39-68, 2001, pp. 59-66).

Se distingue a partir de aquí, la jerarquización de las categorías de análisis y de los componentes ontológicos que intervienen en la relación biología-cultura. Como es característico del esencialismo, la comprensión de las partículas esenciales, que no tienen necesidad de relación alguna para existir, es la condición para comprender el funcionamiento del complejo sistema cultural. En términos epistemológicos esto significa una verticalización jerarquizante de los procedimientos y métodos de 
conocimiento, Hay que concentrarse, en cambio, en la comprensión de los postulados teóricos de la disciplina que estudia a la esencia para entonces lograr comprender a las demás, a las que le están subordinadas y tienen importancia secundaria.

El problema es que así, es imposible comprender al proceso evolutivo, el humano en específico, tal como es, como multidimensionalidad. No se pude comprender que la evolución es el deslizamiento de numerosos planos (Jablonka \& Lamb 2005) que se relacionan, auto-relacionan, cambian y combinan sus jerarquías, sin que exista una preponderancia permanente de uno de esos planos sobre los demás (Levins 2007, p. 149). El análisis de estas interpenetraciones y no las unidireccionalidades, es lo que permite una pluralidad indispensable para la inter y la transdisciplina.

Dada esta multidimensionalidad evolutiva, se debe intentar comprender el conjunto de mediaciones existentes entre el nivel genético y sus manifestaciones exteriores finales, como la cultura y esto en caso de admitir, sin conceder, que lo genético o las "estructuras evolucionadas" -como eufemísticamente les llaman Tooby y Cosmides- fueran realmente uno de los extremos del funcionamiento biológico La comprensión debe hacerse desde una horizontalidad que elimine las jerarquizaciones permanentes y con ello la obligatoriedad de estudiar primero, parcial, disciplinaria y fragmentariamente, las manifestaciones moleculares-hereditarias supuestamente expresadas en los sistemas culturales.

En este trabajo se ha decidido centrar la atención en la obra de Tooby y Cosmides porque se encuentran entre las figuras centrales de la psicología evolutiva y pioneros de la misma, pero es pertinente mencionar que la metodología de esos dos autores no es privativa de ellos en la psicología evolutiva; más bien expresan la generalidad de los casos. Ello se puede encontrar en autores como Buss y Schmidt (1993), Pinker (1997), Thornhill y Palmer (2006), o Bayley, Durante y Geary (2011), para citar sólo a algunos. Ejemplos abundantes de lo afirmado se pueden encontrar en Buss (2005) y Dunbar y Barrett (2007).

\section{Concretos y abstractos}

Este caso de falsa interdisciplina sostiene una metodología que, partiendo de nociones y determinaciones concretas de una realidad, procede a eternizar los procesos descritos yendo de lo concreto a lo abstracto, es decir abstrayendo a estas categorías de sus contextos de relaciones, que son los que producen las determinaciones concretas.

Marx, al referirse al método utilizado por la economía política, explica que contiene una deficiencia dada por confundir lo abstracto con lo concreto, pensar erróneamente que las concreciones, las determinaciones específicas de lo que se ha analizado, no lo son en realidad y que se puede hacer abstracción de ellas y postular esa realidad como realidad universal, indeterminada. Marx defiende que el método correcto debe completarse con el desarrollo ulterior de las categorías abstractas, con la concreción y contextualización de aquello que inicialmente es abstracto, entendido ya como concreto. Al desarrollarse una categoría abstracta, encuentra sus determinaciones concretas en los sistemas de relaciones cambiantes en los que cada una de estas unidades se encuentra. El concepto inicialmente no desarrollado, tendiente a la abstracción; expresa un proceso de evolución, desde luego, a resultas del cual las formas de su expresión y por tanto de su comprensión devienen en concretos más desarrollados, relaciones más subordinadas, multiplicidad de vías o expresiones (Marx [1857-1858] 1987, Tomo I, pp. 20-29)

El movimiento de lo abstracto a lo concreto debe considerar este desarrollo de la categoría. En el problema que nos ocupa, la psicología evolutiva abstrae al gen de sus determinaciones concretas en las que necesita ser comprendido, considerado en este devenir, en la evolución, la multiplicación de sus determinaciones concretas.

Es decir, es un devenir de sus manifestaciones, sus relaciones y la relación es manifestación de lo heterogéneo, de lo diferente, y por esto cada uno de los entes que se analizan, (genes en este caso) son 
ya un tránsito a algo distinto a sí mismos, es el concepto que se desarrolla. En todo ello deviene y codeviene con otros componentes del sistema, se desliza sobre ellos y puede producir otras categorías, otras realidades a las cuales se corresponden otras concreciones, con elementos comunes, pero simultáneamente con otros emergentes. El proceso no se hace en una dirección única. Sus elementos se componen y recomponen, se auto-organizan, sintetizan y degradan; se organizan e interpenetran.

Una epistemología que no refleje y represente adecuadamente estos procesos de diferenciación y negación, estará representando parcialmente esos movimientos ontológicos, manteniendo su comprensión en el nivel de los conceptos no desarrollados, abstractamente considerados como "esenciales". El deslizamiento y las categorizaciones de los fenómenos estudiados se estacionan en un punto que sólo sirve para describir un estado inicial o inicialmente establecido, transmisión de información genotipo-fenotipo en relaciones unitarias causa-efecto.

La psicología evolutiva trabaja con abstracciones concretas despojadas de su devenir y sus contradicciones sus categorías y conceptos no pueden expresar o aplicarse a metodologías interdisciplinarias. Se invagina hacia las expresiones funcionales inmediatas de las categorías que maneja, a sus manifestaciones inmediatas e iniciales, y así, las eterniza.

Bibliografía

Althusser, L. (1988), Ideología y aparatos ideológicos del Estado. Freud y Lacan, Buenos Aires: Nueva Visión.

Bailey, D.H., Durante, K.M. y D.C. Geary (2011), “Men's Perception of Women's Attractiveness is Calibrated to Relative Mate Value and Dominance of the Women's Partner", Evolution and Human Behavior 32: 138-146.

Buss, D.M. y D.P. Schmidt (1993), "Sexual Strategies Theory, An Evolutionary Perspective on Human Mating", Psychological Review 100: 204-232.

Buss, D.M. (eds.) (2005), The Handbook of Evolutionary Psychology, Hoboken: John Wiley \& Sons.

Carnap, R. (1965), "Psicología en lenguaje fisicalista”, en Ayer, A.J. (ed.), El positivismo lógico, México: Fondo de Cultura Económica, pp. 171-204.

Dunbar, M. y L. Barrett (eds.) (2007), The Oxford Handbook of Evolutionary Psychology, Oxford: Oxford University Press.

Engels, F. ([1893] 1997), "Carta a Franz Mehring, 14 de julio de 1893", en Moore, S., Crítica de la Democracia Capitalista, México: Siglo XXI, p. 95.

Farrel, K.N., Luzzeti, T. y S. van den Have (eds.) (2013), Beyond Reductionism, A Passion for Interdisciplinarity, London: Routledge.

Fonkenthal, M. (2001), Interdisciplinarity: Toward the Definition of a Metadiscipline?, New York: Peter Lang.

Frodman, R., Klein, J.T., Mitohuan, C. y J.B. Holbrook (eds.) (2010), The Handbook of Interdisciplinarity, Oxford: Oxford University Press.

García, R. (2007), Sistemas complejos, conceptos, método y fundamentación espistemológica de la investigación interdisciplinaria, México: Gedisa.

Gould, S.J. (1981), La falsa medida del hombre, Barcelona: Antoni Bosch.

Hahn, E. (2000), "Contribución a la crítica de la conciencia burguesa", en Lenk, K. (ed.), El concepto de ideología, Buenos Aires: Amorrortu, pp. 123-136.

Jablonka, E. y M. Lamb (2005), Evolution in Four Dimensions, Genetic, Epigenetic, Behavioral and Symbolic Variation in the History of Life, Cambdidge, MA: MIT Press.

Klein, J.T. (1990), Interdisciplinarity, History, Theory and Practice, Detroit: Wayne State University Press.

Klein, J.T. (1996), Crossing Boundaries: Knowledge, Disciplinarities and Interdisciplinarities, Charlottesville: University Press of Virginia.

Kockelmans, J.J. (1979), Interdiscplinarity and Higher Education, London: The Pennsylvania State University Press. 
Kosick, K. (1967), Dialéctica de lo concreto, México: Grijalbo.

Levins, R. (2007), "Strategies of Abstraction”, en Lewontin, R. y R. Levins, Biology under the Influence: Dialectical Essays on Ecology, Agriculture and Health, New York: Monthly Review Press, pp. 149-166.

Levins, R. y R. Lewontin (1985), "Conclusion, Dialectics", en Levins, R. y R. Lewontin, The Dialectical Biologist, Cambridge, MA: Harvard University Press, pp. 267-288.

Lewontin, R. (1985), “The Organism as Object and Subject of Evolution”, Scientia 118: 63-82. (Reimpreso en Levins, R. y R. Lewontin, The Dialectical Biologist, Cambridge, MA: Harvard University Press, pp. 85-106.)

Lewontin, R. (2000), The Triple Helix, Gene, Organism and Environment, Cambridge, MA: Harvard University Press.

Lewontin, R. (2001), "Gene, Organism and Environment", en Oyama, S., Griffits, P.E. y R.D. Gray (eds.), Cycles of Contingency, Developmental Systems and Evolution, Cambridge, MA: MIT Press, pp. 59-66.

Lewontin, R., Rose, S. y L. Kamin (1984), No está en los genes, racismo, genética e ideología, México: CONACULTA.

Lukacks, G. ([1923]1969), Historia y conciencia de clase, México: Grijalbo.

Lumsden, C.J. y E.O. Wilson (1981), Genes, Mind and Culture. The Coevolutionary Process, Cambridge, MA: Harvard University Press.

Lumsden, C.J. y E.O. Wilson (1983), Promethean Fire: Reflections on the Origin of Mind, Cambridge, MA: Harvard University Press.

Milic, V. (2000), "La relación entre sociedad y conocimiento en la obra de Marx", en Lenk, K. (Comp.), El concepto de ideología, Buenos Aires, Amorrortu, pp. 137-163.

Neurath. O. (1965), "Sociología en fisicalismo”, en Ayer, A.J. (ed.), El positivismo lógico, México: Fondo de Cultura Económica, pp. 287-322.

Newell, W.H. (1990), Interdisciplinarity: Essays from the Literature, New York: College Entumee Examination Board.

Marx, K. ([1845]1953), L’Idéologie Allemande, París: Alfred Costes Editeur.

Marx, K. ([1857-1858] 1987), Elementos fundamentales para la crítica de la economía politica (Grundrisse), México: Siglo XXI.

Ostreng, W. (2010), Science without Boundaries: Interdisciplinarity in Research Society and Politics, London: University Press of America.

Palmer, C. (2000), Work at the Boundaries of Science: Information and the Interdisciplinary Research Process, Dordrecht: Kluwer.

Pichot, A. (2000), La Société Pure. De Darwin a Hitler, Paris: Flammarion.

Pinker, S. (1997), How the Mind Works, London: Penguin.

Ranciêre, J. (1994), “On the Theory of Ideology-Althusser's Politics", en Eagleton, T. (ed.), Ideology, London: Longman, pp. 141-161.

Repko, A.S. (2014), Introduction to Interdisciplinary Studies, Los Angeles: SAGE.

Strober, M.H. (2011), Interdisciplinary Conversations: Challenging the Habits of Thought, Stanford: Stanford University Press.

Szosteck, R. (2010), Classifying Science: Phenomena, Data, Theory, Method, Practice, Dordrecht: Springer.

Thornhill, R. y C.T. Palmer (2006), Una historia natural de la violación. Los fundamentos biológicos de la coerción sexual, México: Océano.

Tooby, J. y L. Cosmides (1992), "The Psychological Foundations of Culture”, en Barkow, J.H., Cosmides, L. y J. Tooby (eds.), The Adapted Mind: Evolutionary Psychology and the Generation of Culture, New York: Oxford University Press, pp. 19-137.

Tooby, J. y L. Cosmides (2005), "Conceptual Foundations of Evolutionary Psychology", en Buss, D.M. (ed.), The Handbook of Evolutionary Psychology, Hoboken, NJ: John Wiley \& Sons, pp. 5-68.

Trivers, R. (1971), “The Evolution of Reciprocal Altruism”, The Quarterly Review of Biology 46: 45-57. 
96 | Julio Muñoz Rubio

Weingart, P. y N. Stehr (eds.) (2000), Practicing Interdisciplinarity, Toronto: University of Toronto Press.

Wilson, E.O. (1975), Sociobiology, The New Synthesis, Cambridge, MA: Harvard University Press.

Wilson, E.O. (1978), On Human Nature, Cambridge, MA: Harvard University Press.

Young, R.M. (1973), “The Historiographical and Ideological Context of the Nineteenth Century Debate on Man's Place in Nature”, en Teich, M. y R.M. Young (eds.), Changing Perspectives in the History of Science, Dordrecht: Reidel, pp. 344-438.

Zizek, S. (1994), “CĆmo inventó Marx el síntoma?”, en Zizek, S. (ed.), Ideología. Un Mapa de la Cuestión, México: Fondo de Cultura Económica, pp. 329-370. 